\title{
Discovering items with potential popularity on social media
}

\author{
Khushnood Abbas',201414060110@std.uestc.edu.cn \\ Shang Mingsheng ${ }^{2}$, msshang@cigit.ac.cn \\ LuoXin ${ }^{3}$,luoxin21@cqu.edu.cn \\ 1. Web science center University of Electronic science and Technology Of China (UESTC) \\ 2. Chongqing Institute of Green and Intelligent Technology, Chinese Academy of \\ Sciences(CIGIT) \\ 3. Chongqing University Chongqing China
}

\begin{abstract}
Predicting the future popularity of online content is highly important in many applications. Preferential attachment phenomena is encountered in scale free networks. Under it's influece popular items get more popular thereby resulting in long tailed distribution problem. Consequently, new items which can be popular (potential ones), are suppressed by the already popular items. This paper proposes a novel model which is able to identify potential items. It identifies the potentially popular items by considering the number of links or ratings it has recieved in recent past along with it's popularity decay. For obtaining an effecient model we consider only temporal features of the content, avoiding the cost of extracting other features. We have found that people follow recent behaviours of their peers. In presence of fit or quality items already popular items lose it's popularity. Prediction accuracy is measured on three industrial datasets namely Movielens, Netflix and Facebook wall post. Experimental results show that compare to state-of-the-art model our model have better prediction accuracy.
\end{abstract}

\section{Introduction}

E-commerce and social-media offer their users facilities to buy, review, sell and share online items, such as Amazon, e-Bay, Facebook and Tencent QQ. The adoption of social media is increasing by day and night so as e-commerce. Because of its widely adoption, it is gaining attention of the organizations, to know people's demand and trend.Researchers have also found correlation between social media and e-commerce industry. Social media is showing a great opportunity by carrying huge amount of user generated data for exploiting and finding the outcome of interest (Asur, Huberman, and others 2010). In e-commerce site, organizations are directly interested what are the response from users, for their products. They also want to know which kind of item will be in demand so that they can make profit. Since the number of products are expanding constantly so online merchants changes their strategies from traditional marketing advertisement (like TV, news paper etc) to viral marketing, i.e. customers are suggested to share the product information with their friends on social media such as Facebook and twitter (Leskovec, Adamic, and Huberman 2007). From infotainment to trade, everything is done on Internet and online contents have become valuable internet asset (Tatar et al. 2014) that is useful to producers and consumers both, hence to know the future popularity of online content has become an important area of attention and interest.

Popularity prediction is a complex task depending on different factors like quality and individual's interest.Content popularity may fluctuate with time (Eisler, Bartos, and Kertész 2008), increase over time or be limited within communities. It is difficult to capture the relationship between real world event and web content, in the prediction model for example during Indian election many joke goes viral and they might not explicity inlude the political symbol.Some contents get extreme popularity because of its prior popularity, also known as cascading effect (Cheng et al. 2014), and it becomes hard to predict which content will stop this cascading effect. In presence of cascading effect other "potential items" (exhibit a sudden increase of 
popularity at a certain duration of time, i.e. can be popular, but not now) are suppressed.Most recently researchers have found that the popularity of online contents like news, blog posts, videos, mobile app download (Gleeson et al. 2014) in online discussion forums and product reviews exhibits temporal dynamics. It turns out that user interests toward web items, vary with time; an extensive study of how the popularity of online media's temporal patterns grow and fade, over time are presented in (Yang and Leskovec 2011), (Leskovec, Backstrom, and Kleinberg 2009). Besides the works on temporal scale, other researchers found that the contents popularity is influenced significantly by consumers social relationship (ZENG et al. 2013), (Szabo and Huberman 2015), the social network of consumer can enhance the prediction performance; and social dynamics of consumer, influence the social media content's popularity even more significantly. Because the models of both temporal dynamics and social dynamics are always complex and parameterized, it is hard to apply those models to real online systems.

The rest of the paper is organized as follows. In Section 2 we formally defined problem. In section 3 we introduced the baseline method. Insection 4 we proposed new method to solve the problem. In section 5 we discuss the mthods and materials for experiment and we also discuss insights from the results.In Section 6 we have concluded the paper with possible future works.

\section{Problem definition}

In our model we have considered the bipartite network which consists of a set of users $(U)$ and a set of objects $(O)$. The popularity of a node or item or object is the total link recieved by the object $(o)$. A bipartite network can be represented by an adjacency matrix $A$, if user $u,(u \in U)$ have consumed object $o,(o \in O)$. To consider the temporal effect on objects' final popularity we take snapshots of the network at different time point. Let $A(t)$ denote the adjecency matrix of the snap-shotted network at time $t$, then matrix $(A(t))$ containes edges between user $(u)$ and object $(o)$ before time $(t)$ only. The user and object degree can be computed by $k_{u}(t)=\sum_{o} A_{u o}(t)$ and $k_{o}(t)=\sum_{u} A_{u o}(t)$, respectively. $T_{F}$ is future time window, so popularity or increment in degree of an object $o$ is given by-

$$
\Delta k_{o}\left(t, T_{F}\right)=k_{o}\left(t, T_{F}\right)-k_{o}(t)
$$

The popularity prediction problem can be defined as follows. Given a data-set $\mathrm{S}$ which includes information about ratings by users that have rated or consumed the item/object $o$ with time-stamp (e.g. user id, item id, time), after arranging the data-set in ascending order by time, we divided the data-sets into training time $\mathrm{t}$ and future time window $T_{F}$. This is obvious that interest towards object, varies with type or category such as online video, object is different than items on Amazon. Considering these facts we have chosen the training time $t$ that helps us to predict with better accuracy in future time window. A predictor exploits the information before time $(t)$ and make prediction for future time window $\left(T_{F}\right) . \Delta k_{o}\left(t, T_{F}\right)$ is the real score of object $(o)$. A predictor's performance is measured by calculating it's accuracy by comparing its ranking from predicted ranking and real ranking.

\section{Baseline method for comparison}

We have considered state-of-the art for predicting new entries as a baseline method as for as our knowledge goes.

\section{Popularity-based predictor}

(ZENG et al. 2013) has proposed Popularity based predictors(PBP). It is based on a well known preferential attachment theory, which states that popularity increases cumulatively; the rate of new link (Either item recieves rating in case of Movielens, or a friend like or comments in case of Facebook wall post activity) 
formation for any node is proportional to the observed number of links which node has recieved in past. If an item is popular at time $t$, then it will probably become popular due to the condition that current degree of an item $\mathrm{k}_{o}(\mathrm{t})$ is a good predictor of its future popularity. Further ((Gleeson et al. 2014), (ZENG et al. 2013)) have found that current degree is a good predictor of items' future popularity.(ZENG et al. 2013) proposes to calculate the prediction score of an item at time $t$ can be given as follows- -

$$
\mathrm{s}_{o}\left(\mathrm{t}, \mathrm{T}_{\mathrm{p}}\right)=\mathrm{k}_{o}(\mathrm{t})-\lambda \mathrm{k}_{o}\left(\mathrm{t}, \mathrm{T}_{\mathrm{P}}\right)
$$

Where $\Delta \mathrm{k}_{o}\left(\mathrm{t}, \mathrm{T}_{\mathrm{P}}\right)$ is the rating/links recieved in past time window $T_{P}$ from $t . \lambda \in[0,1]$, note that $\lambda=0$ gives the total popularity and for $\lambda=1$ it gives recent popularity. Throught the script by popularity we mean number of ratings or links recieved by item or node.

\section{Proposed method: Considering aging factor with recent popularity.}

It is obvious that popularity of any item on social media doesn't last forever.In addition decay rate may vary from item to item e.g life cycle of popularity of a movie will be different from news or items on Digg or Facebook.Every type of item have its own decay rate like (Parolo et al. 2015) have found research article citation rate decays after some time. Mathew effect or preferential attachement is a well known phenomena seen almost in large scale networks that shows power law distribution (et.al 1999). These theories explains that rich will get richer and poor get poorer.

As researchers have found that degree distribution of every network ends up to long tailed.This is also true in case of e-commerce user-item bipartite networks.People chase the popularity of items to optimize their time and energy. There are good or fit items (Matus Medo Manuel S. Mariani and Zhang 2015), (Bianconi and Barab $\tilde{A}_{i s i}$ 2001) to be consumed but under the influence of preferential attachment those are ignored. Under it's influence finding the fit or potential item is one of the important tasks among the researchers.Researchers have also found that network changes structure due to aging factor over time [(H. Zhu, Wang, and Zhu 2003)]. Considering these factors we propose that the recent gain in popularity as well as decay in popularity together are a good predictor for its future popularity.Considering the competing behavior in networks (Bianconi and Barab $\widetilde{A}_{j}$ si 2001), if some items are loosing their populairty then other items should be gaining attentions of the consumer. Therefore decaying factors with recent popularity will help us in detecting "potantial items". Recent popularity is one of the important factors in discovering the final popularity of objects are discussed in (J.-P. Onnela and Reed-Tsochas 2010). We also know that considering all features that affect popularity of content is really a difficult task. If $\mathrm{s}_{o}\left(\mathrm{t}, \mathrm{T}_{\mathrm{p}}\right)$ is prediction score at time $t$ given past time window $T_{P}$. We can say-

$$
s_{o}\left(t, T_{p}\right) \prec \sum_{u}\left(k_{o}(t)-\lambda k_{o}\left(t-T_{P}\right)\right)
$$

The above equation states that score of object is proportional to recent gain in popularity. $\lambda$ is tunable prameter between recentness and total popularity.it can take values in $[0,1]$ interval. As the researchers also found aging phenomina in item or node so we can formulate it as follows-

$$
s_{o}\left(t, T_{p}\right) \prec \sum_{u} e^{\gamma\left(T_{u_{o}}-t\right)}
$$

Where $T_{u o}$ denotes the time at which user $u$ consumed the object $o$ and $\gamma$ is free parameter.Since recent popularity will be good predictor if decay rate is constant. So now we can write as follows-

$$
s_{o}\left(t, T_{p}\right) \prec \sum_{u}\left(k_{o}(t)-\lambda k_{o}\left(t-T_{P}\right)\right) * e^{\gamma\left(T_{u o}-t\right)}
$$


again we can write-

$$
s_{o}\left(t, T_{p}\right)=l \sum_{u}\left(k_{o}(t)-\lambda k_{o}\left(t-T_{P}\right)\right) * e^{\gamma\left(T_{u o}-t\right)}
$$

where $l$ is normalization constant and can be estimated using following equation $\sum_{o} s_{o}\left(t, T_{p}\right)=1$.

\section{Experimental Results and discussion}

For testing our proposed predictor's efficiecy we have considered Popularity Based Predictor (PBP) as a base predictor by (ZENG et al. 2013). We took average of 10 results.

\section{Evalutation metrics}

Three evaluation metrics are adopted to measure the accuracy of the proposed model including preci$\operatorname{sion}\left(P_{n}\right)$,novelty $\left(Q_{n}\right)$ and Area Under Recieving Operating Characteristic $(A U C)$.

- Precision is defined as the fraction of objects that are predicted also lie in the top $N$ object of true ranking (Herlocker et al. 2004).

$$
p_{n}=\frac{D_{n}}{n}
$$

Where $D_{n}$ is the number of common objects between predicted and real ranking. $n$ is the size of list to be ranked.It's value ranges in $[0,1]$, higher value of $\left(P_{n}\right)$ is better.

- $\operatorname{Novelty}\left(Q_{n}\right)$ is a metric to measure the ability of a predictor to rank the items in top $n$ position that was not in top $n$ position in previous time window. We call these new entries as "potential items" throughout the script. If we denote the predicted object as $\left(P_{p} o\right)$ and potential true object as $P_{r} o$, then the novelty of a model is given by-

$$
Q_{n}=P_{p o} / P_{r o}
$$

- $A U C$ measures the relative position of the predicted item and true ranked items. Suppose predicted item list is $\left(L_{p} n\right)$ and real item list is $\left(L_{r} n\right)$. if $s_{o} p \in L_{p n}$ and $s_{r} p \in L_{r n}$ is score of object in predicted then $A U C$ is given by-

$$
A U C=\frac{\sum_{o p \in L_{p n}} \sum_{r p \in L_{r n}} I\left(s_{p n}, s_{r n}\right)}{\left|L_{p n}\right|\left|L_{r n}\right|}
$$

where,

$$
I\left(s_{p n}, s_{r n}\right)=\left\{\begin{array}{c}
0 \Leftarrow s_{p n}<s_{r n} \\
0.5 \Leftarrow s_{p n}<s_{r n} \\
1 \Leftarrow s_{p n}>s_{r n}
\end{array}\right.
$$




\section{Data used in this article}

To test the predictors accuracy we have used different data sets. Like MovieLens, Netflix, Facebook wall post datasets etc. MovieLens and netflix data sets contain movie ratings and Facebook data set contains users' wall post relationships. MovieLens is provided by GroupLens project at University of Minnesota. The data description can be found on the website. While data preparation for our model we have selected small subset from each by randomly choosing users who have rated atleast 20 movies. The original rating was in the form of numarical $1-5$, we have considered the link between the user and object which object have recieved higher than two ratings.For all the three datasets Facebook, Movielens and Netflix the time is considered in days. The data description is as follows-

- Netflix data contains 4960 users,16599 movies and 1249058 links, data was collected during(1st Jan $2000-31$ st Dec 2005).

- MovieLens dataset contains 7533 movies, 864581 links and 5000 users and data was collected during(1st Jan $2002-1$ st Jan 2005).

- Facebook data contains 40981 set of users and their 38143 wall post activity and 855542 links, during period of (14 Sep 2004-22nd Jan 2009). If user has posted on a wall there will be a link between the user and the wall, self influenced is removed by removing the link between user and its own wall post.

\section{Accuracy results on different datasets}

To evaluate the performance of our predictors we have selected 10 random $t$ for each data sets. Selection of $\mathrm{t}$ is considered in such a way that predictor have enough history information. Since predictors are based on objects' history, we have selected only those object that have recieved atleast one link before time $t$.
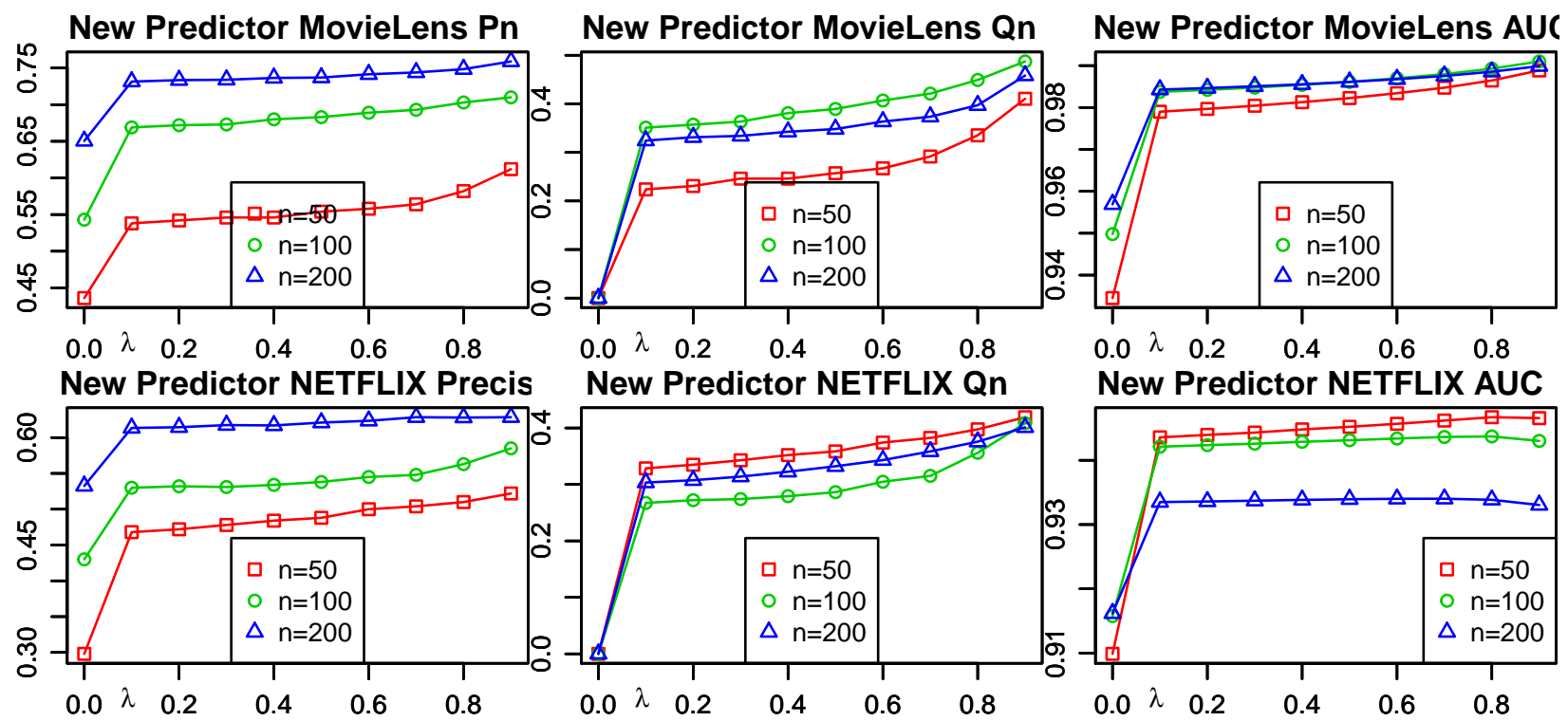

New Predictor NETFLIX Qn
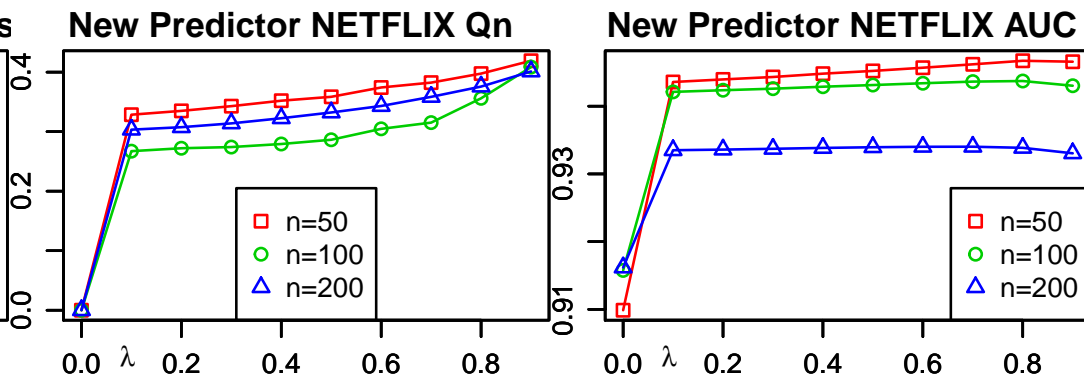

New Predictor Facebook Pn

New Predictor Facebook Qn
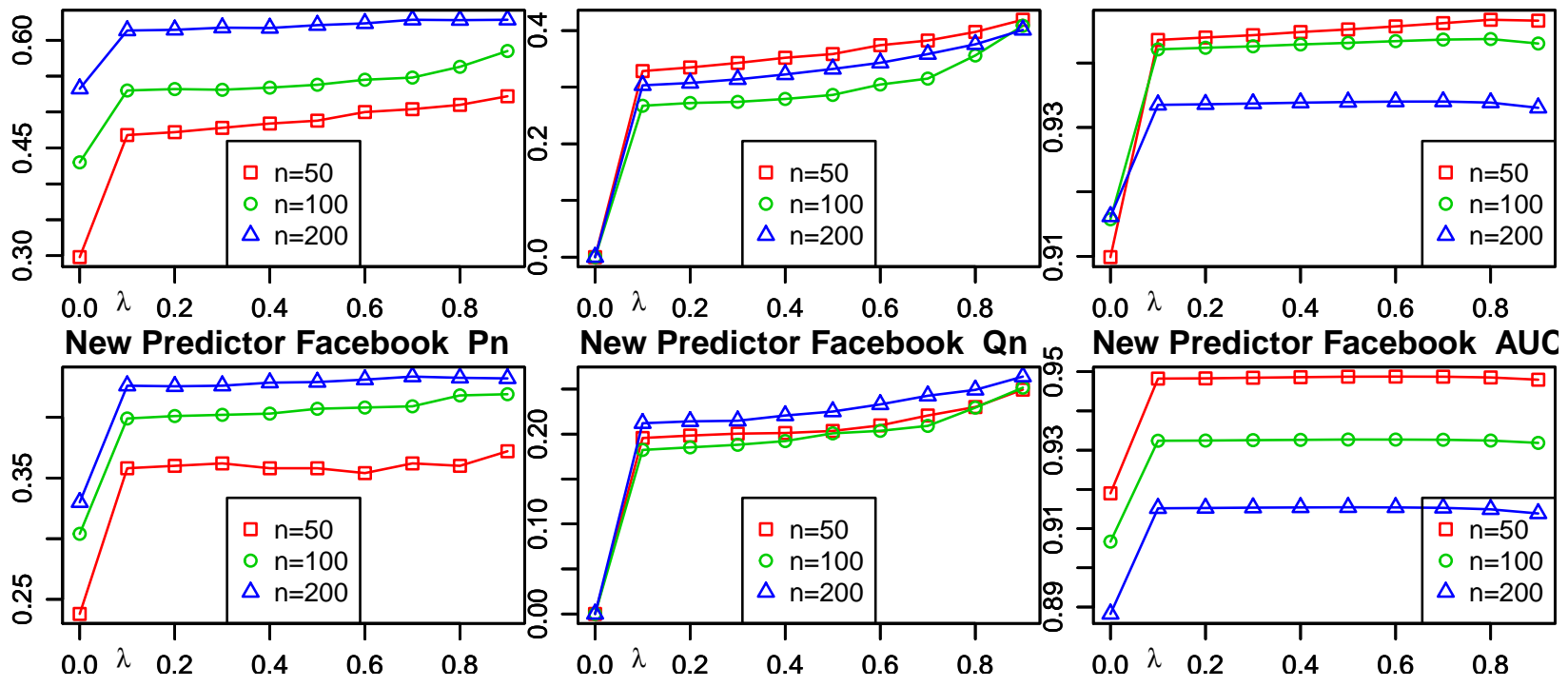
Figure 1: The performance of the proposed method for different values of $n$ (Top $\mathrm{n}$ items in a list). For Three data sets Movielens, Netfix and Facebook wall post.

In the above [Figure 1] we have shown the proposed predictors performance under different values of $\lambda$, i.e considering the recent popularity with variying history lenght because $\lambda=0$ means objects total popularity and $\lambda=1$ means objects recent popularity. We have consider the decay rate $\gamma$ as fix, because we have found $\gamma=0.1$ gives better result.From the figure [Figure 1] we can see $P_{n}$ is much affected by $n$ than $Q_{n}$ (novelty) and $A U C$. All the three metrics improves with $\lambda$ in other words recent populairty is a good predictor than the object's total popularity. Higher $P_{n}$ values shows that proposed predictor have better ability to predict popularity of objects than the base method while our predictor have ability to predict better the popularity of novel items $\left(Q_{n}\right)$,i.e the items that were not popular in the past time. These items are "potential items", these items will help in abating the centrality of item's degree distribution. Generally these items are suppressed by items that have already gained populairty. Our proposed predictor have also shown improvment over the base method.

\section{Effect of aging Vs recent popularity}

In decay rate $(\gamma)$ and recent popularity analysis we have found decay rate is very low for all the three datasets. Considering decay with recent populartiy improves accuracy. We have also found considering decay helps more in digging new entries. Although aging factor improves the accuracy but still recent behavior dominates. We have also found that in the presence of quality item people lose interest in old items that is why $Q_{n}$ improves when considering decay factor with recent poularity.Even if the item is not globally popular people like the items that were liked by the peers recently. Empirical results are as follows-

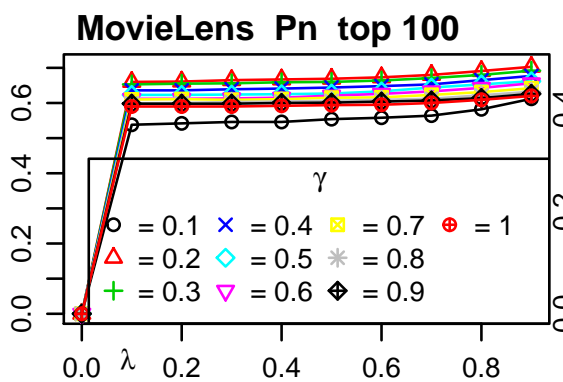

MovieLens Qn top 100

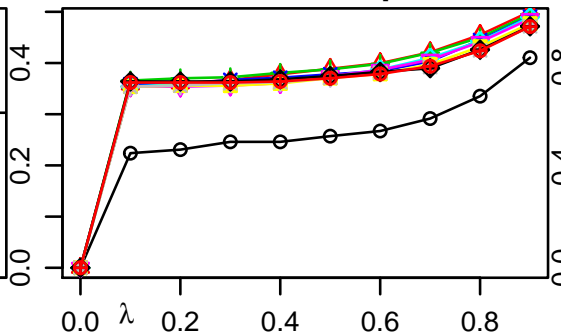

Netflix Qn top 100
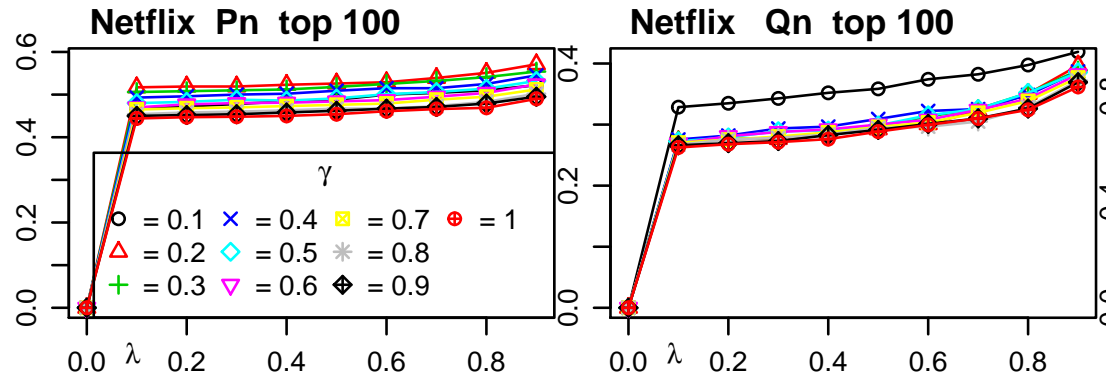

Facebook Pn top 100

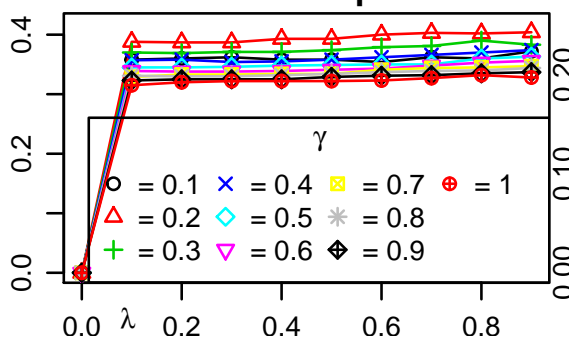

Facebook Qn top 100

MovieLens AUC top 100

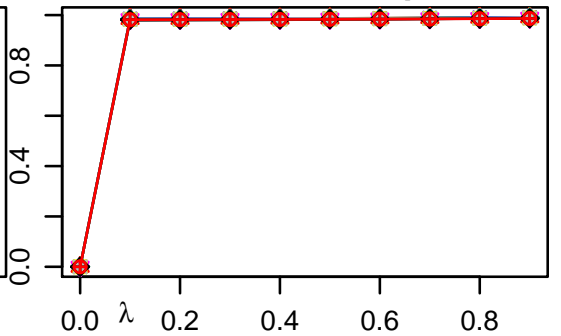

Netflix AUC top 100

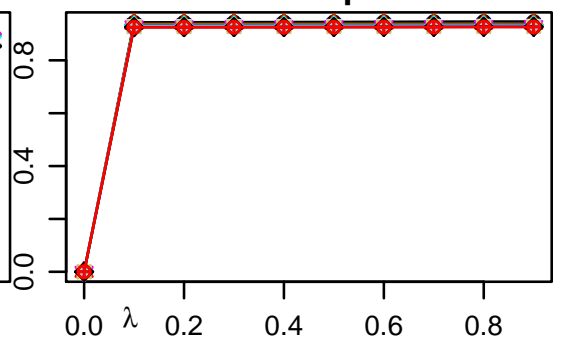

Facebook AUC top 100

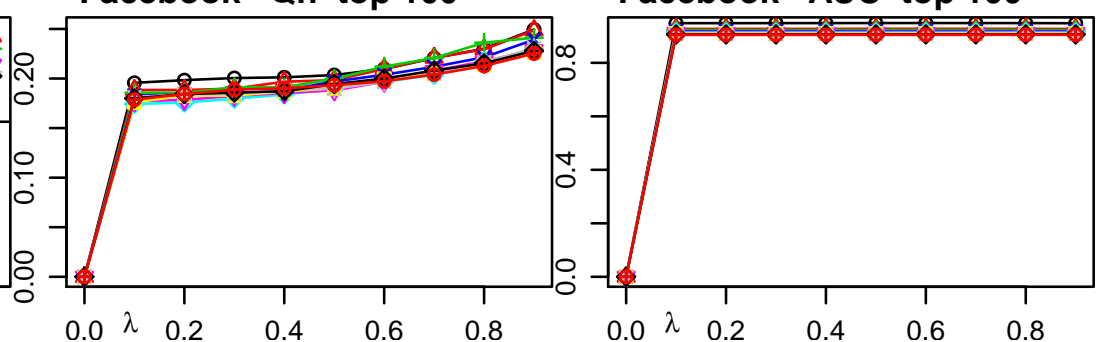

Figure 2: The performance of our proposed predictor for different values of $\lambda$ and $\gamma$ (decay rate). We have considered $T_{F}$ and $T_{P}$ both as 30 days. 
In search of our predictor's behavior we have considered different values of $\gamma$ and plotted [Figure 2] the accuracy against $\lambda$. Although $\gamma$ is free parameter but to know its effect we considered 10 values in $[0,1]$. We have tried recentness of edge collection by the node with decay rate to see the effect together. We have found popular items last longer on Movielens since precision is better for $\gamma=0.2$. We have found people adopts new items from recent behaviours of their peers. In other words for trying new things they rely on their peer's behaviour. Since $Q_{n}$ not much affected by decay rate as compare recent behaviours. In cas of Netflix we found the same nature as Movielens popular items last for long time. In case of Facebook we have found that people rely more on the age of the post than the recent behaviours of their friends. While more recent post shared by their friends are more entertained.On Facebook not only share or comment on their friend's post but also they can create their own post and other can share or comment.That is why potantial items on Facebook, a user not only depends on the age and recent activity of the peers but also node cetrality. Who have shared the post also matters. That is why predicting new popular items on Facebook needs more feature consideration such as centrality of the node,time etc.

The [Table 1] gives detailed comparison of the two predictors. We have considered future time window as well as past time window both as 30 days. The second column is accuracy of our proposed predictor while PBP column is the base predictor. The three numbers in both the columns are basically for top 50,100 and 200 items respectively from left to right. The "Type" column describe the accuracy type, every accuracy is compared for three cases : $\mathrm{n}=50,100$ and 200 items.It is easy to see that proposed predictor has shown improvement.

\section{Comparison of two predictors}

For comparing our proposed predictor with base predictor we have considered past time window $\left(T_{P}\right)$ and future time window $\left(T_{F}\right)$ as 30 days. For comparison we have selected the top n ranked items from predicted list and compare them against the real items for both the predictors. Emprical results are as follows-

Table 1: Perfomrance table for both the predictor considering Tp and $\mathrm{Tf}$ as 30 days. The volues are for top 50,100 and 200 items respectively.

\begin{tabular}{llll}
\hline DataSet & Proposed 50/100/200 & PBP 50/100/200 & Type \\
\hline MovieLens & $0.612,0.71,0.759$ & $0.498,0.646,0.721$ & Pn \\
Netflix & $0.522,0.585,0.628$ & $0.462,0.534,0.609$ & Pn \\
Facebook & $0.372,0.419,0.434$ & $0.358,0.398,0.426$ & Pn \\
MovieLens & $0.41,0.487,0.458$ & $0.126,0.253,0.243$ & Qn \\
Netflix & $0.419,0.408,0.401$ & $0.302,0.263,0.267$ & Qn \\
Facebook & $0.249,0.252,0.264$ & $0.19,0.177,0.207$ & Qn \\
MovieLens & $0.989,0.991,0.99$ & $0.976,0.982,0.982$ & AUC \\
Netflix & $0.947,0.944,0.934$ & $0.943,0.942,0.936$ & AUC \\
Facebook & $0.949,0.933,0.915$ & $0.947,0.933,0.916$ & AUC \\
\hline
\end{tabular}

\section{Accuracy comparison}

We have compared our results with the base method considering top 50,100,200 list. For comparing we have considered training windown $\left(T_{P}\right)$ as 30 days and we have tested the predictor for the same future time lenght $T_{F}=30$ days. 

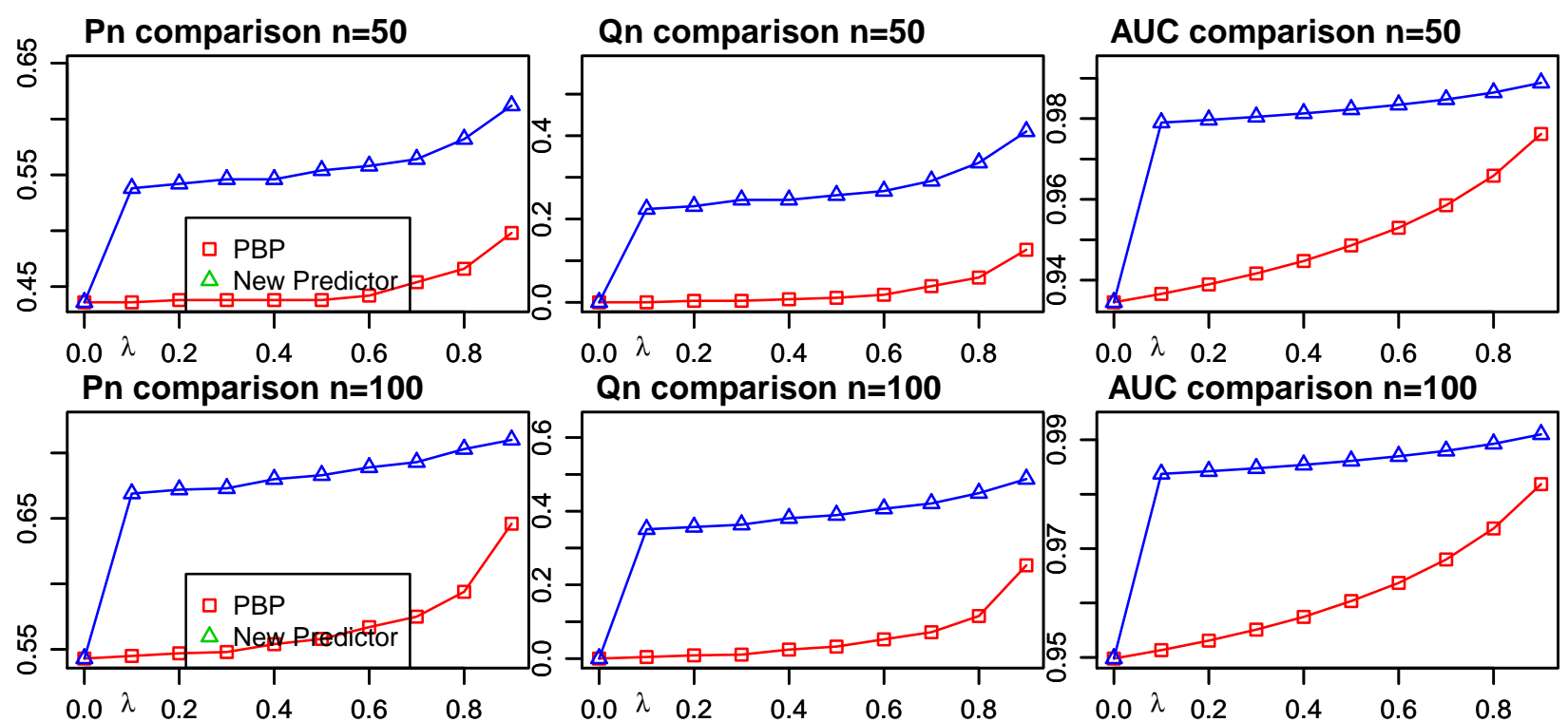

Qn comparison $n=100$

AUC comparison $n=100$
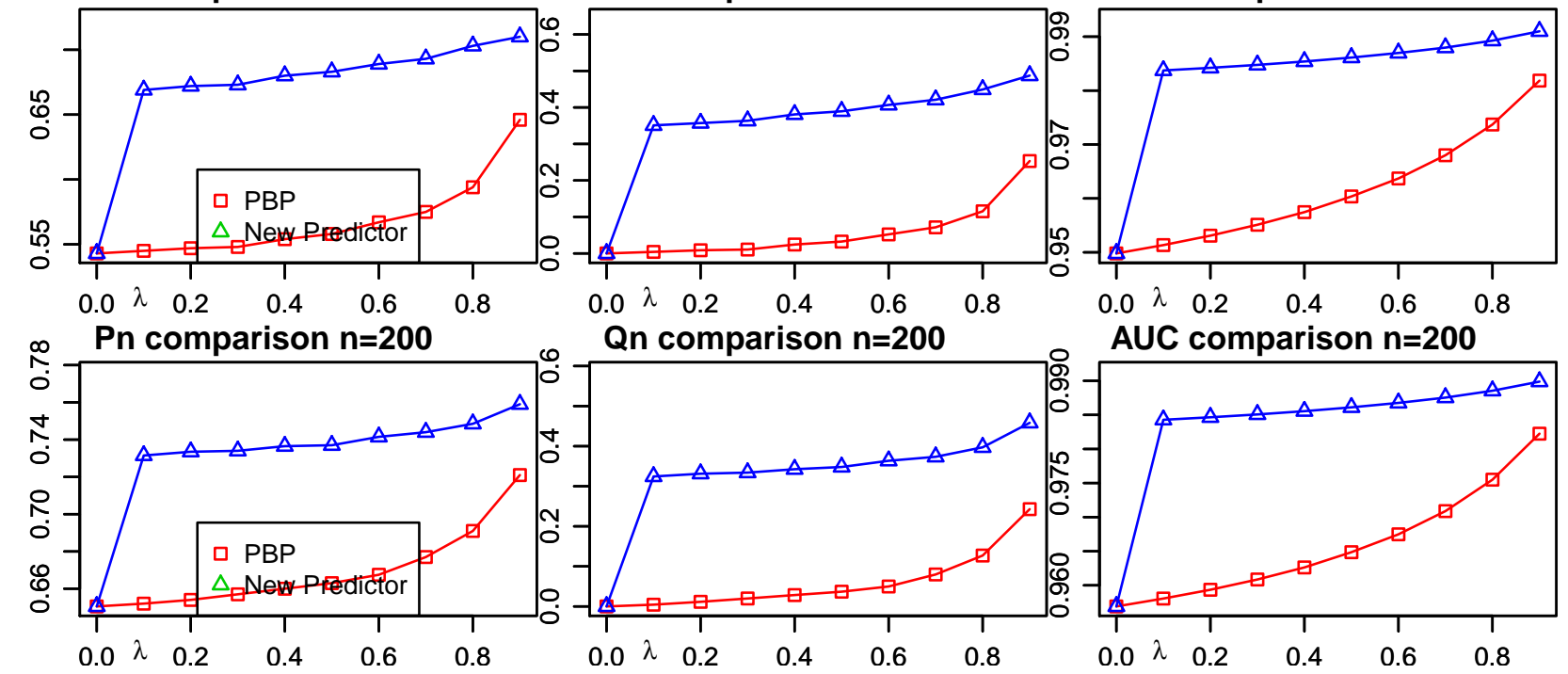

AUC comparison $n=200$

Figure 3: The performance comparison between proposed method and base method for MovieLens dataset. For the above comparison we have considered $T_{F}=30$ and $T_{P}=30$ days.

[Figure 3,Figure 4,Figure 5] show the comparitive performence of proposed method over base method. It is easy to find that our proposed method out performs the base method in all the situation. For all the datasets $P_{n}$ is better for all values of $n . Q_{n}$ also out performs as compare to the base predictor. If the item has low time span, the prediction made by total popularity is not good while the prediction made by recent popularity method for the same is good.Results also show using recent popularity works good for all the datasets; MovieLens, Netflix and Facebook, and for all the situations. When the future window length is short.Prediction by total popularity works good in case of item has already gained long term popularity. 


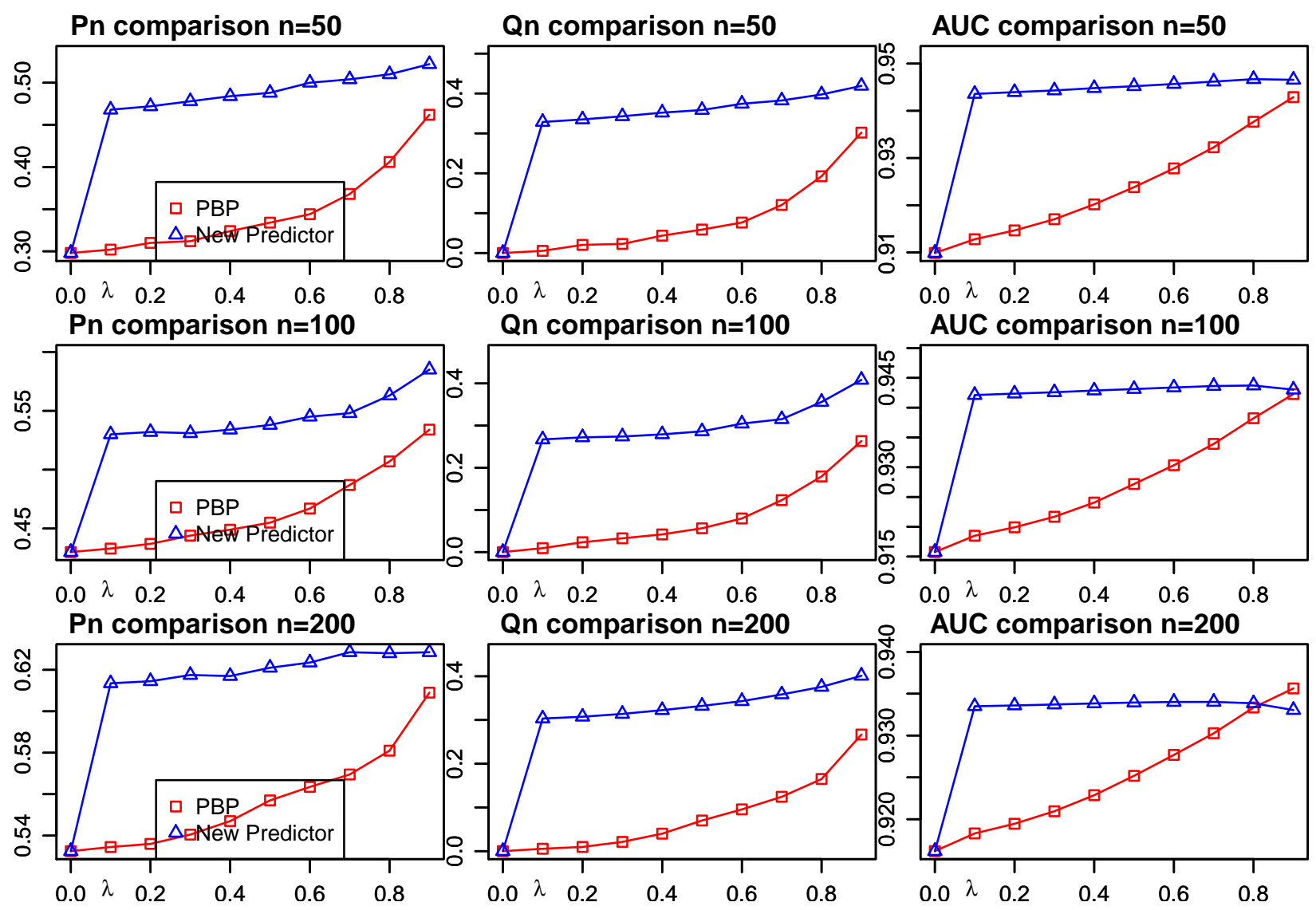

Figure 4: The performance comparison between proposed method and base method for Netflix dataset. For the above comparison we have considered $T_{F}=30$ and $T_{P}=30$ days.

Charecteristric of all the three datasets are not same, such as Facebook content may not be alive after few weeks while on Movielens and NetFlix content may never die. Further more the rate at which node attract new links also differ such as the most popular node will recieve more attention than less popular ones. The content on Facebook may not be always intersting to friends so that they can share with their friends such as friends may not like content on politics. While content on Movielens and Netflix are appealing to viewers. That is the reason our predictor's accuracy for Facebook dataset as not good as Movielens and Netflix. In [Figure 6] we find precision get better with $\lambda$ for all the datasets suggest that people like the item that their peers are watching or liking in recent time. 


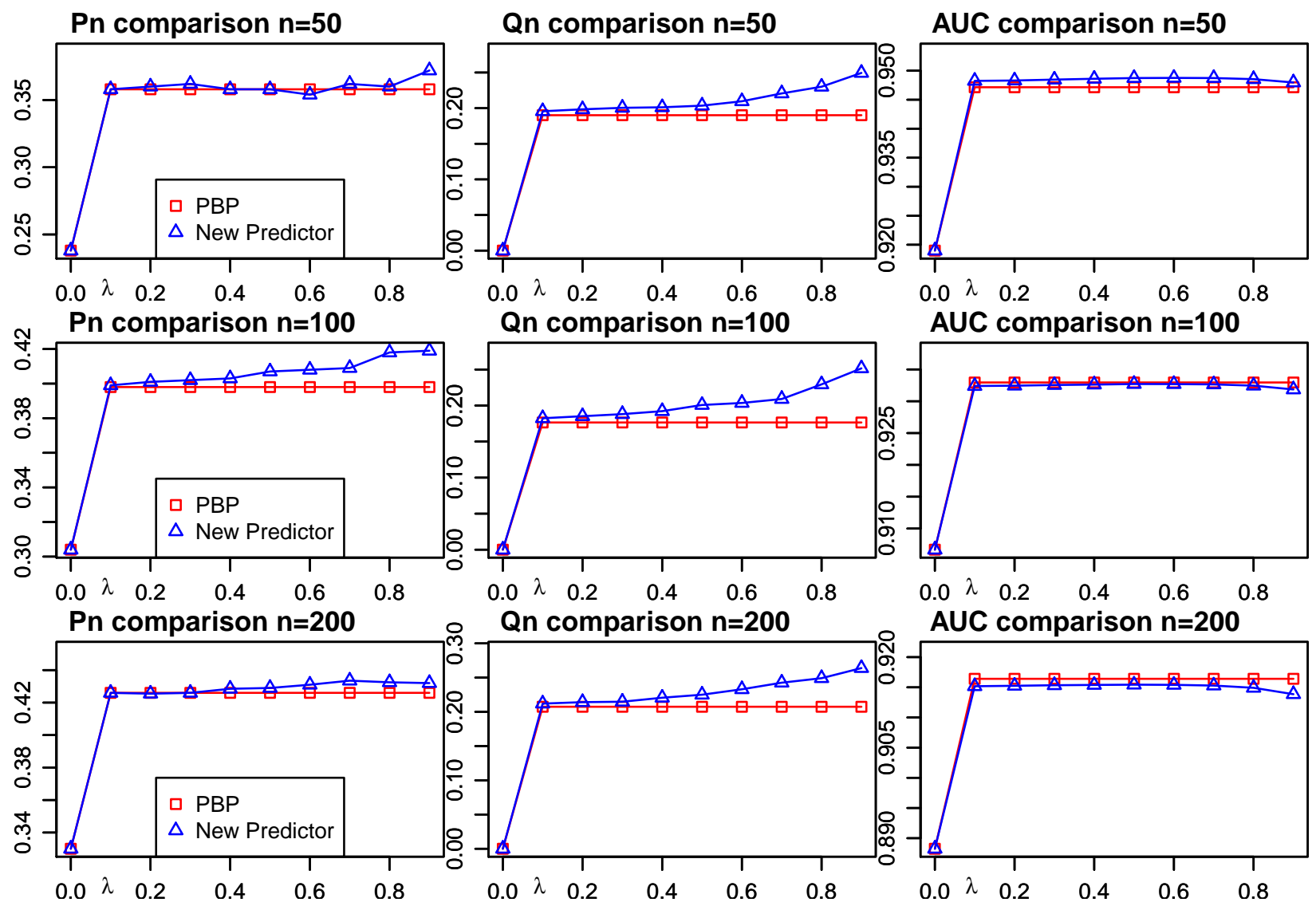

Figure 5: The performance comparison between proposed method and base method for Facebook. For the above comparison we have considered $T_{F}=30$ and $T_{P}=30$ days.

Our predictor also outperforms the base method (based on recent popularity) also shows that content popularity are affected by it's age also since we have considered aging in our model. As aging phenomena is present in every network therefore new entries get chance to become popular. We can think in two ways, either due to aging of old popular items new entries get attention or due to quality or fitness of item. If node achieve popularity due to its fitness suggest it is showing competitive behaviour.Scientist have found both the phenomena in real networks. In our case we can argue new entries become popular not only because old entries aging effect but also items fitness because there are so many entries to watch or consumed. If people are watching or liking any item it is because of it's innate quality not because they dont have enough entries to watch or consume. We know there are plenty of new entries available for all the cases namely Movielens,Netflix and Facebook. Therefore we can say that in the presence of quality item they attract link from the popular item to become popular by showing competitve behaviour.

\section{Predictor's perfomance for varying future time lenght $T_{F}$}

In [Figure 7] we have shown the performance of our predictor against the base predictor for different values of future time window.For Proposed predictor $\lambda=0.9$ and $\gamma=0.1$ for PBP $\lambda=0.9$ and past time window length $T_{P}=60$ days as the author has used in his paper. It is easy to discover that smaller $T_{F}$ lenght helps in predicting for short time while long $T_{F}$ helps for predicting long term trend. 

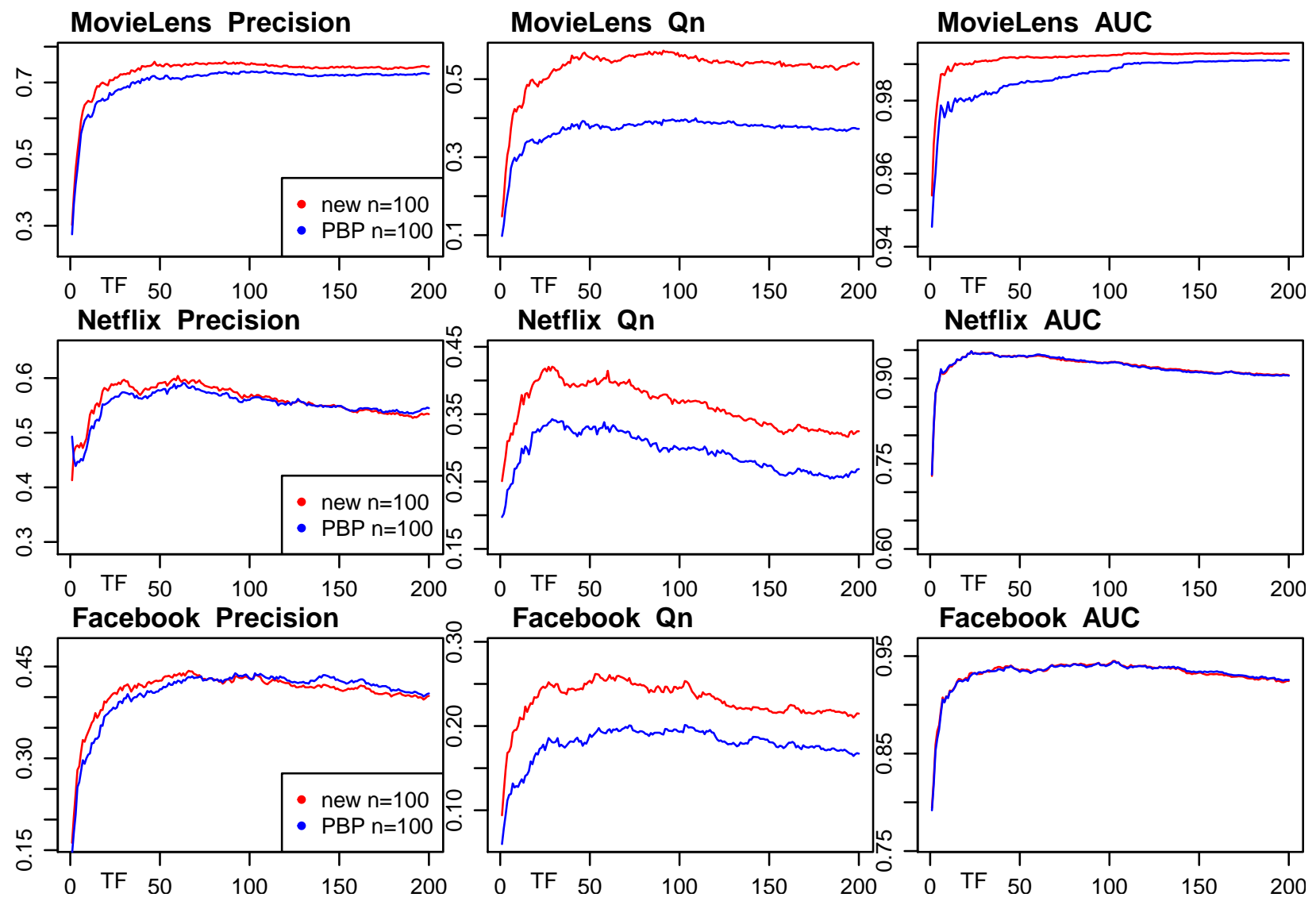

Facebook Qn

Facebook AUC
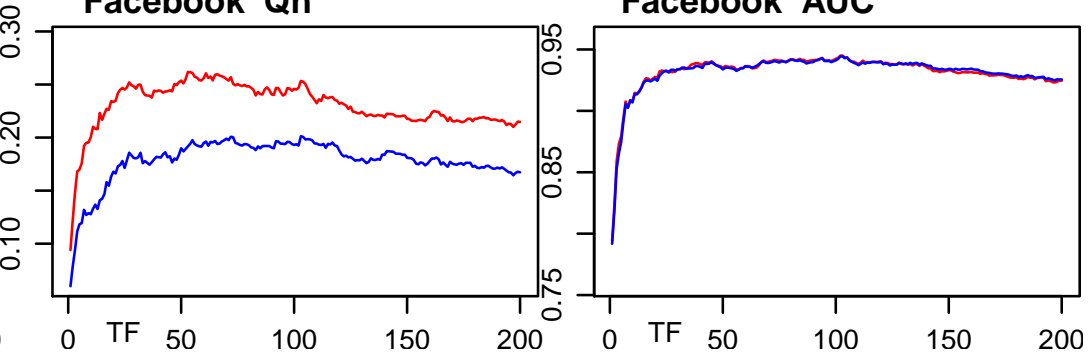

Figure 7: The above figure shows the performance of the predictor for diferent values on future time window $T_{F}$. Red line shows the performance of our proposed predictor while blue shows the base PBP predictor.

We have chosen the $T_{P}$ same as $T_{F}$ in case of PBP the author has considered. As we can see from the [Figure 7] our proposed predictor has better performance in predicting the long term trend prediction. We have considered the future time window for 200 days to evaluate the performance of our predictor. We have found that in case of Movielens people tend to copy the behaviours of their peers as well as they also like to explore new entries.In case of Netflix we have found precision shows improvement for around 100 days but after that PBP performed better. Although in enquiry of novel items our predictor is better. This phenomena also suggest that people explore new itmes on Netlix and also rely on their peer's recent activity. Facebook activity data shows similar nature. Our predictor shows significant precision for upto 200 days. It also predicts the behaviour of consumers for exploring or trying "new thing" nature. Our works describe presence "potantial items" those are generally subdued in the presence of other already popular items.

\section{Conclusion}

In this manuscript we came up with one model to make prediction of object on online social media specially considering it's temporal behavior. We created a model by considering object's recent popularity as well as it's aging or decay of popularity.Epirical results show that our proposed method out performs the base method i.e popularity based predictor given by (ZENG et al. 2013). We have found that people tend to copy recent behaviours of their peer consumers not the whole popularity of items. We have also found that in presence of quality items recent popular items loses it's popularity or we can say that on these kinds of network competitive behavior (described by (Bianconi and Barab $\tilde{A}_{j}$ si 2001) for social network) also found.We have 
considered only temporal effects of the node's attracting new link.We have found it one of the important feature for making prediction.In future work one can also consider other effects like human dynamics,item category,node centrality etc.

\section{References}

Asur, Sitaram, Bernardo Huberman, and others. 2010. "Predicting the Future with Social Media." In Web Intelligence and Intelligent Agent Technology (WI-IAT), 2010 IEEE/WIC/ACM International Conference on, edited by IEEE, 1:492-99. IEEE. doi:10.1109/WI-IAT.2010.63.

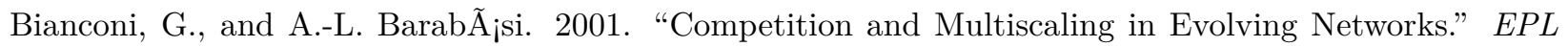
(Europhysics Letters) 54 (4): 436. http://stacks.iop.org/0295-5075/54/i=4/a=436.

Cheng, Justin, Lada A. Adamic, P. Alex Dow, Jon M. Kleinberg, and Jure Leskovec. 2014. "Can Cascades Be Predicted?" In 23rd International World Wide Web Conference, Www '14, Seoul, Republic of Korea, April 7-11, 2014, edited by Chin-Wan Chung, Andrei Z. Broder, Kyuseok Shim, and Torsten Suel, 925-36. ACM. doi:10.1145/2566486.2567997.

Eisler, Zoltán, Imre Bartos, and János Kertész. 2008. "Fluctuation Scaling in Complex Systems: Taylors Law and Beyond1." Advances in Physics 57 (1). Informa UK Limited: 89-142. doi:10.1080/00018730801893043.

et.al, A. Barabasi. 1999. "Emergence of Scaling in Random Networks." Science 286 (5439). American Association for the Advancement of Science (AAAS): 509-12. doi:10.1126/science.286.5439.509.

Gleeson, James P, Davide Cellai, Jukka-Pekka Onnela, Mason A Porter, and Felix Reed-Tsochas. 2014. "A Simple Generative Model of Collective Online Behavior." Proceedings of the National Academy of Sciences 111 (29). National Acad Sciences: 10411-15. doi:10.1073/pnas.1313895111.

Herlocker, Jonathan L., Joseph A. Konstan, Loren G. Terveen, and John T. Riedl. 2004. "Evaluating Collaborative Filtering Recommender Systems." ACM Transactions on Information Systems 22 (1). Association for Computing Machinery (ACM): 5-53. doi:10.1145/963770.963772.

Leskovec, Jure, Lada A Adamic, and Bernardo A Huberman. 2007. "The Dynamics of Viral Marketing." ACM Transactions on the Web (TWEB) 1 (1). ACM: 5. doi:10.1145/1232722.1232727.

Leskovec, Jure, Lars Backstrom, and Jon M. Kleinberg. 2009. "Meme-Tracking and the Dynamics of the News Cycle." In Proceedings of the 15th ACM SIGKDD International Conference on Knowledge Discovery and Data Mining, Paris, France, June 28 - July 1, 2009, edited by John F. Elder IV, Françoise Fogelman-Soulié, Peter A. Flach, and Mohammed Javeed Zaki, 497-506. ACM. doi:10.1145/1557019.1557077.

Matus Medo Manuel S. Mariani, An Zeng, and Yi-Cheng Zhang. 2015. "Identification and Modeling of Discoverers in Online Social Systems." http://arxiv.org/pdf/1509.01477.pdf. http://arxiv.org/pdf/1509. 01477.pdf.

Onnela, J.-P., and F. Reed-Tsochas. 2010. "Spontaneous Emergence of Social Influence in Online Systems." Proceedings of the National Academy of Sciences 107 (43). Proceedings of the National Academy of Sciences: 18375-80. doi:10.1073/pnas.0914572107.

Parolo, Pietro Della Briotta, Raj Kumar Pan, Rumi Ghosh, Bernardo A. Huberman, Kimmo Kaski, and Santo Fortunato. 2015. "Attention Decay in Science." J. Informetrics 9 (4): 734-45. doi:10.1016/j.joi.2015.07.006.

Szabo, Gabor, and Bernardo A. Huberman. 2015. "Predicting the Popularity of Online Content." Communications of the ACM. http://doi.acm.org/10.1145/1787234.1787254. doi:10.1145/1787234.1787254.

Tatar, Alexandru, Marcelo Dias de Amorim, Serge Fdida, and Panayotis Antoniadis. 2014. "A Survey on Predicting the Popularity of Web Content." J Internet Serv Appl 5 (1). Springer Science Business Media. doi:10.1186/s13174-014-0008-y. 
Yang, Jaewon, and Jure Leskovec. 2011. "Patterns of Temporal Variation in Online Media." In Proceedings of the Fourth ACM International Conference on Web Search and Data Mining, edited by ACM, 177-86. WSDM '11. New York, NY, USA: ACM. doi:10.1145/1935826.1935863.

ZENG, AN, STANISLAO GUALDI, MATÚŠ MEDO, and YI-CHENG ZHANG. 2013. "Trend Prediction in Temporal Bipartite Networks: The Case of Movielens, Netglix, and Digg." Advances in Complex Systems 16 (04n05). World Scientific Pub Co Pte Lt: 1350024. doi:10.1142/s0219525913500240.

Zhu, Han, Xinran Wang, and Jian-Yang Zhu. 2003. "Effect of Aging on Network Structure." Physical Review E 68 (5). American Physical Society (APS). doi:10.1103/physreve.68.056121. 